\title{
SÍNDROMES HEREDITARIOS DE CÁNCER GASTROINTESTINAL: EL ROL DE LA ASESORÍA
} GENÉTICA

HEREDITARY GASTROINTESTINAL CANCER SYNDROMES: THE ROLE OF GENETIC COUNSELING SINDROMES DO CANCRO GASTROINTESTINAL HEREDITÁRIO: O PAPEL DO ACONSELHAMENTO GENÉTICO

\section{Castro-Mujica, María del Carmen ${ }^{1}$, Abarca-Barriga, Hugo²}

\author{
1 Médica genetista. Sequence Reference Lab. Facultad de Medicina Humana - Universidad Ricardo Palma. Lima, Perú. \\ Email de contacto: mcastro@sequence.pe \\ 2 Médico Genetista. Facultad de Medicina Humana - Universidad Ricardo Palma. Lima, Perú.
}

\section{Estimado Director:}

Sr. Editor, La Red Nacional Integral del Cáncer (NCCN) ha determinado que, ante la sospecha de un síndrome de predisposición al cáncer, es necesario referir al paciente a evaluación genética debido a las implicancias en el diagnóstico y manejo, así como el seguimiento en sus familiares (1). La evaluación genética incluye la descripción de los antecedentes personales/familiares y la solicitud del estudio genético correspondiente.

El Síndrome Lynch (SL) es responsable del 3\% del total de casos de cáncer colorrectal (CCR) y es considerado el síndrome genético más frecuente en CCR (2). Los pacientes con variantes en los genes MMR tienen un riesgo de hasta $82 \%$ a desarrollar CCR (2). Por otro lado, los síndromes hereditarios de poliposis colónica se clasifican según la edad de presentación, tipo y número de pólipos, patrón de herencia, genes asociados, manifestaciones extracolónicas y el riesgo a desarrollar CCR (2). Estos incluyen principalmente a: Poliposis adenomatosa familiar (PAF), PAF atenuada, poliposis asociado a MUTYH, síndrome de poliposis juvenil, síndrome Peutz-Jeghers y síndrome Cowden, principalmente (Tabla 1).

En relación al cáncer gástrico, sólo un 3\% son de origen genético (3). El cáncer gástrico difuso hereditario (CGDH), debido a variantes en el gen $\mathrm{CDH} 1$, se caracteriza por conferir un riesgo de $67 \%$ y $83 \%$ a desarrollar cáncer gástrico en varones y mujeres, respectivamente. Estos casos se presentan, usualmente, antes de los 40 años de edad y son multifocales (3).

Finalmente, alrededor del $10 \%$ del total de casos de cáncer de páncreas están asociados a diversos síndromes genéticos como (4): Síndrome Peutz-Jeghers, síndrome FAMMM, pancreatitis hereditaria, síndrome de cáncer mama/ovario hereditario, síndrome VHL (Tabla 1). Así mismo, se han descrito otros genes relacionados a la susceptibilidad a desarrollar cáncer de páncreas: PALB2, PALLD, ATM.

Las guías clínicas han establecido los seguimientos necesarios en los casos antes mencionados $(1,5)$. En SL se ha considerado que las colonoscopías anuales deben iniciarse a la edad de 20-25 años, mientras que en PAF debe ser a los 10-12 años de edad. Con respecto al CGDH, se recomienda realizar endoscopía alta anual desde la adolescencia y considerar la gastrectomía total profiláctica después de los 20 años de edad. En relación a los casos de predisposición al cáncer de páncreas, se ha establecido controles con ultrasonido endoscópico alternando con colangiopancreatografía por resonancia magnética anual desde los 50 años de edad.

La evaluación y asesoría genética es una estrategia costo-efectiva para identificar a los pacientes con síndromes de predisposición al cáncer gastrointestinal y establecer el manejo multidisciplinario a fin de prevenir y/o detectar oportunamente el cáncer, reduciendo su morbimortalidad en el paciente y familiares.

\begin{tabular}{|c|c|c|c|}
\hline SÍNDROME $\quad$ HEREDITARIO & DE|GEN(ES) & RIESGO DE CÁN & RRIESGO DE OTROS \\
\hline PREDISPOSICIÓN AL CÁNCER & & GASTROINTESTINAL & TIPOS DE CÁNCER \\
\hline Síndrome Lynch & $\begin{array}{l}\text { MLH1 } \\
\text { MSH2 } \\
P M S 2 \\
\text { MSH6 } \\
\text { EPCAM }\end{array}$ & \begin{tabular}{|l|} 
Colorectal \\
Intestino delgado \\
Estómago \\
Tracto biliar \\
Páncreas
\end{tabular} & $\begin{array}{l}\text { Endometrio } \\
\text { Ovario } \\
\text { Pelvis renal y ureter } \\
\text { SNC } \\
\text { Mama } \\
\text { Tumores sebáceos }\end{array}$ \\
\hline Poliposis adenomatosa familiar & $A P C$ & $\begin{array}{l}\text { Colorectal } \\
\text { Duodeno } \\
\text { Estómago } \\
\text { Hepatoblastoma }\end{array}$ & $\begin{array}{l}\text { Tiroides (papilar) } \\
\text { Meduloblastoma } \\
\text { Adrenal }\end{array}$ \\
\hline
\end{tabular}




\begin{tabular}{|c|c|c|c|}
\hline & & Tumor desmoide & \\
\hline Poliposis adenomatosa & \multirow[t]{2}{*}{$A P C$} & Colorectal & \multirow[t]{2}{*}{ Tiroides (papilar) } \\
\hline familiar atenuada & & Duodeno & \\
\hline \multirow[t]{4}{*}{ Poliposis asociado a MUTYH } & \multirow[t]{4}{*}{ MUTYH } & Colorectal & Ovario \\
\hline & & Duodeno & Vejiga \\
\hline & & & Mama \\
\hline & & & Endometrio \\
\hline \multirow[t]{4}{*}{ Síndrome de Poliposis Juvenil } & SMAD4 & Colorectal & - \\
\hline & $B M P R 1 A$ & Intestino delgado & \\
\hline & & Estómago & \\
\hline & & Páncreas & \\
\hline \multirow[t]{4}{*}{ Síndrome Peutz-Jeghers } & \multirow[t]{4}{*}{ STK11 } & Colorectal & Mama \\
\hline & & Intestino delgado & Cérvix \\
\hline & & Estómago & Ovario \\
\hline & & Páncreas & Testicular \\
\hline $\begin{array}{l}\text { Poliposis asociada a la corrección de } \\
\text { polimerasa }\end{array}$ & $\begin{array}{l}\text { POLE } \\
\text { POLD1 }\end{array}$ & Colorectal & Endometrio \\
\hline \multirow[t]{4}{*}{ Síndrome Cowden } & \multirow[t]{4}{*}{ PTEN } & \multirow[t]{4}{*}{ Colorectal } & Mama \\
\hline & & & Tiroides (no medular) \\
\hline & & & Renal \\
\hline & & & Endometrio \\
\hline \multirow{2}{*}{ Cáncer gástrico difuso hereditario } & \multirow[t]{2}{*}{$\mathrm{CDH} 1$} & Estómago (difuso) & \multirow[t]{2}{*}{ Mama (Iobulillar) } \\
\hline & & Colorectal & \\
\hline \multirow[t]{6}{*}{ Síndrome Li-Fraumeni } & \multirow[t]{6}{*}{ TP53 } & Colorectal & Mama \\
\hline & & Estómago & Sarcoma \\
\hline & & Páncreas & Leucemia \\
\hline & & & SNC \\
\hline & & & Adrenocortical \\
\hline & & & Próstata \\
\hline \multirow[t]{3}{*}{$\begin{array}{l}\text { Síndrome de melanoma familiar con } \\
\text { lunares atípicos múltiples (FAMMM) }\end{array}$} & \multirow[t]{3}{*}{$C D K N 2 A$} & Páncreas & Melanoma malign \\
\hline & & & Pulmón \\
\hline & & & Mama \\
\hline \multirow{3}{*}{$\begin{array}{l}\text { Síndrome } \\
\text { hereditario }\end{array}$} & \multirow{3}{*}{$\begin{array}{l}B R C A 1 \\
B R C A 2\end{array}$} & \multirow[t]{3}{*}{ Páncreas } & Mama (mujer/varón) \\
\hline & & & Ovario \\
\hline & & & Próstata \\
\hline \multirow[t]{2}{*}{ Pancreatitis hereditaria } & PRSS1 & \multirow[t]{2}{*}{ Páncreas } & - \\
\hline & SPINK1 & & \\
\hline Síndrome Von Hippel-Lindau & $V H L$ & Páncreas & Renal \\
\hline \multirow[t]{3}{*}{ Susceptibilidad al cáncer de páncreas } & PALB2 & \multirow[t]{3}{*}{ Páncreas } & \multirow{3}{*}{$\begin{array}{l}\text { Diversos tipos de cáncer } \\
\text { (según gen) }\end{array}$} \\
\hline & PALLD & & \\
\hline & ATM & & \\
\hline
\end{tabular}

Palabras claves: Asesoramiento Genético; Síndromes Neoplásicos Hereditarios; Neoplasias Gastrointestinales

\section{Bibliografía}

1. NCCN. Genetic/Familial High-Risk Assessment: colorectal. NCCN Clinical Practice Guidelines in Oncology. 2019

2. Castro M, Barletta C. Síndrome de Lynch: aspectos genéticos, clínicos y diagnósticos. Rev Gastroenterol Peru. 2018 Jul-Sep;38(3):265-279. Review.

3. Colvin H, Yamamoto K, Wada N, Mori M. Hereditary gastric cancer syndromes. Surg Oncol Clin N Am 2015;24(4):765-777.

4. Connor AA, Gallinger S. Hereditary pancreatic cancer syndromes. Surg Oncol Clin N Am 2015;24(4):733-764.

5. Syngal S, Brand RE, Church JM, et al. ACG clinical guideline: genetic testing and management of hereditary gastrointestinal cancer sydromes. Am J Gastroenterol 2015;110(2):223-62

Recibido: 2020-11-26

DOI: http://dx.doi.org/ 10.31053/1853.0605.v77.n4.26534

(c) Universidad Nacional de Córdoba

(c) (i) (8) 